\title{
DUALITY OF WEIGHTED BERGMAN SPACES WITH SMALL EXPONENTS
}

\author{
Antti Perälä and Jouni Rättyä \\ University of Eastern Finland, Department of Physics and Mathematics \\ P. O. Box 111, FI-80101 Joensuu, Finland; antti.perala@uef.fi \\ University of Eastern Finland, Department of Physics and Mathematics \\ P. O. Box 111, FI-80101 Joensuu, Finland; jouni.rattya@uef.fi
}

\begin{abstract}
It is shown that for each radial doubling weight $\omega$ and $0<p<1$, the dual of the weighted Bergman space $A_{\omega}^{p}$ can be identified with the Bloch space under the $A_{W}^{2}$-pairing, where $W$ satisfies a two sided doubling property and depends on both $\omega$ and $p$. This result generalizes the duality relation $\left(A_{\alpha}^{p}\right)^{*} \simeq \mathcal{B}$, studied by Shapiro, Coifman, Rochberg and Zhu, concerning the classical weighted Bergman spaces.
\end{abstract}

\section{Introduction and the result}

Let $\mathcal{H}(\mathbf{D})$ denote the space of analytic functions in the unit disc $\mathbf{D}$. An integrable function $\omega: \mathbf{D} \rightarrow[0, \infty)$ is called a weight. It is radial if $\omega(z)=\omega(|z|)$ for all $z \in \mathbf{D}$. For $0<p<\infty$ and a radial weight $\omega$, the weighted Bergman space $A_{\omega}^{p}$ consists of $f \in \mathcal{H}(\mathbf{D})$ such that

$$
\|f\|_{A_{\omega}^{p}}^{p}=\int_{\mathbf{D}}|f(z)|^{p} \omega(z) d A(z)<\infty .
$$

As usual, we write $A_{\alpha}^{p}$ if $\omega(z)=\left(1-|z|^{2}\right)^{\alpha}$ for $-1<\alpha<\infty$. When $p \geqslant 1$, the space $A_{\omega}^{p}$ is a Banach space, while for $p<1$ the expression above defines only a quasi-norm, under which $A_{\omega}^{p}$ is complete. In this work, we will study the continuous dual of $A_{\omega}^{p}$, denoted by $\left(A_{\omega}^{p}\right)^{*}$, consisting of all linear maps $F: A_{\omega}^{p} \rightarrow \mathbf{C}$ such that $|F(f)| \leqslant C\|f\|_{A_{\omega}^{p}}$ with $C>0$ independent of $f$. Note that $\left(A_{\omega}^{p}\right)^{*}$ is always a Banach space, even for $p<1$, when the space $A_{\omega}^{p}$ itself is not.

It is classical that in the case of standard weights the dual of $A_{\alpha}^{p}$ is isomorphic to $A_{\alpha}^{p^{\prime}}$ when $p>1$, and $\left(A_{\alpha}^{1}\right)^{*} \simeq \mathcal{B}$. Here $\mathcal{B}$ denotes the Bloch space that consists of $f \in \mathcal{H}(\mathbf{D})$ such that

$$
\|f\|_{\mathcal{B}}=\sup _{z \in \mathbf{D}}\left|f^{\prime}(z)\right|\left(1-|z|^{2}\right)+|f(0)|<\infty
$$

For $p>1$ and through a similar argument for $p=1$, the duality can be proven roughly as follows. Take element $F \in\left(A_{\alpha}^{p}\right)^{*}$, and extend it by the Hahn-Banach theorem to an element of the dual of $L_{\alpha}^{p}$. Now, due to the existence of an isometric isomorphism $\left(L_{\alpha}^{p}\right)^{*} \simeq L_{\alpha}^{p^{\prime}}$ with $1 / p+1 / p^{\prime}=1$, we find $g_{F} \in L_{\omega}^{p^{\prime}}$ representing $F$. Finally, we project $g_{F}$ to $A_{\omega}^{p^{\prime}}$ and use the self-adjointness to get the desired duality. Now, if $p<1$, using the above approach one immediately runs into trouble. First, $A_{\alpha}^{p}$ is not locally convex, so the Hahn-Banach theorem fails to hold altogether. Even

https://doi.org/10.5186/aasfm.2017.4239

2010 Mathematics Subject Classification: Primary 30H20; Secondary 46A16.

Key words: Bergman projection, Bergman space, Bloch space, Carleson measure, doubling weight, duality, reproducing kernel estimate.

This research was supported in part by Academy of Finland project no. 268009 and Ministerio de Economía y Competitivivad, Spain, project MTM2014-52865-P. 
if one blindly assumes that Hahn-Banach holds, another obstruction presents itself, namely $\left(L_{\alpha}^{p}\right)^{*}=\{0\}$. This clearly cannot be the way to proceed, as $\left(A_{\alpha}^{p}\right)^{*}$ is easily seen to contain non-zero elements, such as the point-evaluations. In [10] Zhu showed that for $0<p<1$, the duality $\left(A_{\alpha}^{p}\right)^{*} \simeq \mathcal{B}$ still holds under an appropriately chosen dual pairing depending on $\alpha$. In fact, as Zhu mentions, this result was obtained for the Bergman space $A^{p}$ in 1976 by Shapiro [9], and for the weighted Bergman space $A_{\alpha}^{p}$ by Coifman and Rochberg [1] a few years later. The Hardy space case was first dealt with by Romberg in his $1960 \mathrm{PhD}$ thesis [8]. In this work, we will obtain analogous duality result for a class of Bergman spaces generated by much more general weights. To state the result and to place it in a more general context, some notation and background is needed. Write $\omega \in \widehat{\mathcal{D}}$ if $\widehat{\omega}(z)=\int_{|z|}^{1} \omega(t) d t$ satisfies the doubling property $\widehat{\omega}(r) \leqslant C \widehat{\omega}\left(\frac{1+r}{2}\right)$ for all $r \in[0,1)$. All the standard weights $\left(1-|z|^{2}\right)^{\alpha}$ belong to $\widehat{\mathcal{D}}$, but this doubling property is also satisfied by the weights $(1-|z|)^{-1}\left(\log \frac{e}{1-|z|}\right)^{-\beta}$ for $\beta>1$ that induce essentially smaller Bergman spaces than the standard weights. If $\omega \in \widehat{\mathcal{D}}$ and there exists $K=K(\omega)>1$ and $C=C(\omega)>1$ such that $\widehat{\omega}(r) \geqslant C \widehat{\omega}\left(1-\frac{1-r}{K}\right)$ for all $0 \leqslant r<1$, then we denote $\omega \in \mathcal{D}$. For basic properties of these weights and more, see [3], [6] and [7].

If $\omega \in \mathcal{D}$, then $\left(A_{\omega}^{p}\right)^{*} \simeq A_{\omega}^{p^{\prime}}$ for $1<p<\infty$, and $\left(A_{\omega}^{1}\right)^{*} \simeq \mathcal{B}$ by [5, Corollary 7]. The reason why the proof of the first-mentioned result does not carry over the whole class $\widehat{\mathcal{D}}$ is that the maximal Bergman projection

$$
P_{\omega}^{+}(f)(z)=\int_{\mathbf{D}} f(\zeta)\left|B_{z}^{\omega}(\zeta)\right| \omega(\zeta) d A(\zeta)
$$

where $B_{z}^{\omega}$ stands for the reproducing kernel of the Hilbert space $A_{\omega}^{2}$, is not necessarily bounded on $L_{\omega}^{p}$ for all $\omega \in \widehat{\mathcal{D}}$ by [5, Theorem 5]. Therefore as far as we know, the existing literature does not offer a description of the dual of $A_{\omega}^{p}$ when $\omega \in \widehat{\mathcal{D}}$. It is also worth mentioning that it is not known if the Bergman projection $P_{\omega}$ is bounded on $L_{\omega}^{p}$ if $\omega \in \widehat{\mathcal{D}} \backslash \mathcal{D}$ and $1<p<\infty$ excepting of course the trivial case $p=2$.

In this work we describe the dual of $A_{\omega}^{p}$ for $0<p<1$ and $\omega \in \widehat{\mathcal{D}}$. To define the appropriate dual pairing we define for $\omega \in \widehat{\mathcal{D}}$ and $0<p \leqslant 1$, the weight

$$
W(z)=W_{p, \omega}(z)=\left(\frac{1}{p}-1\right) \widehat{\omega}(z)^{\frac{1}{p}}(1-|z|)^{\frac{1}{p}-2}+\frac{\omega(z)}{p} \widehat{\omega}(z)^{\frac{1}{p}-1}(1-|z|)^{\frac{1}{p}-1}, \quad z \in \mathbf{D} .
$$

Then $\widehat{W}(z)=\widehat{\omega}(z)^{\frac{1}{p}}(1-|z|)^{\frac{1}{p}-1}$, and, in particular, $W_{1, \omega}=\omega$. One could equally well consider any of the summands appearing in the definition of $W$ only, but because of the identity for $\widehat{W}$ we keep this definition since it slightly simplifies some calculations that we will face later. For $r \in(0,1)$, define $f_{r}$ by $f_{r}(z)=f(r z)$ for all $z \in \mathbf{D}$. With these preparations we can state the main result of this study as follows.

Theorem 1. Let $0<p<1$ and $\omega \in \widehat{\mathcal{D}}$. Then $\left(A_{\omega}^{p}\right)^{*} \simeq \mathcal{B}$, with equivalence of norms, under the pairing

$$
\langle f, g\rangle_{A_{W}^{2}}=\lim _{r \rightarrow 1^{-}} \int_{\mathbf{D}} f_{r}(z) \overline{g(z)} W(z) d A(z), \quad f \in A_{\omega}^{p}, \quad g \in \mathcal{B} .
$$

Comparing with Zhu's argument [10], we are led to deal with the following three main obstructions:

(1) $L^{p}$-estimates for functions resembling the Bergman kernel;

(2) Sharp embedding $A_{\omega}^{p} \subset A_{W}^{1}$; 
(3) Fractional differential operators.

In the case of the standard weights, (1) is taken care of by the classical Forelli-Rudin estimates [2], while the requirement (2) can be understood in terms of Carleson measures. It turns out, that these lie in the core of the theory of more general weights, and indeed can be understood. As far as we know, there is no direct analog for the fractional differential operators induced by more general weights; for standard weights the order of differentiation comes out rather transparently. Surprisingly enough, we can avoid fractional derivatives altogether, and this actually simplifies the proof substantially.

\section{Towards Theorem 1}

Zhu's argument goes through expressing $f \in A_{\omega}^{p}$ with the help of the reproducing property. Our reasoning could be considered a somewhat more streamlined version of this proof. It relies in particular on [5, Theorem 1(ii)] which plays the role of the Forelli-Rudin estimates and says that, for each $\omega, \nu \in \widehat{\mathcal{D}}, 0<p<\infty$ and $n \in \mathbf{N} \cup\{0\}$, the reproducing kernel of $A_{\omega}^{2}$ satisfies the estimate

$$
\left\|\left(B_{z}^{\omega}\right)^{(n)}\right\|_{A_{\nu}^{p}}^{p} \asymp \int_{0}^{|z|} \frac{\widehat{\nu}(t)}{\widehat{\omega}(t)^{p}(1-t)^{p(n+1)}} d t, \quad|z| \rightarrow 1^{-} .
$$

This result was used in [5] to show that for each $\omega \in \mathcal{D}$, the spaces $\left(A_{\omega}^{1}\right)^{*}$ and $\mathcal{B}$ are isomorphic via the pairing

$$
\langle f, g\rangle_{A_{\omega}^{2}}=\lim _{r \rightarrow 1^{-}} \int_{\mathbf{D}} f_{r}(z) \overline{g(z)} \omega(z) d A(z), \quad f \in A_{\omega}^{1}, \quad g \in \mathcal{B} .
$$

The above result can be used to prove the surjectivity of $P_{\omega}: L^{\infty}(\mathbf{D}) \rightarrow \mathcal{B}$. The proof is quite standard, but not readily available in the literature, so we record it here for the convenience of the reader. To find an exact preimage of $g \in \mathcal{B}$ under $P_{\omega}$ is more laborious, see [7] for details. Here we just note that if $\omega \in \mathcal{D}$ satisfies the pointwise regularity condition $\widehat{\omega}(z) \asymp \omega(z)(1-|z|)$, then for each $g \in \mathcal{B}$, the function

$$
h(z)=g(0)+\frac{\widehat{\omega}(z)}{|z| \omega(z)}\left(2 z g^{\prime}(z)+g(z)-g(0)\right)
$$

belongs to $L^{\infty}$ and satisfies $P_{\omega}(h)=g$.

Lemma 2. Let $\omega \in \mathcal{D}$. Then $P_{\omega}: L^{\infty} \rightarrow \mathcal{B}$ is bounded and onto.

Proof. The fact that $P_{\omega}: L^{\infty} \rightarrow \mathcal{B}$ is bounded for each $\omega \in \widehat{\mathcal{D}}$ is contained in [5, Theorem 5(ii)], but since the proof is short we sketch it here for the convenience of the reader. If $h \in L^{\infty}$, then $\left(P_{\omega}(h)^{\prime}(z)\right)=\int_{\mathbf{D}} h(\zeta)\left(B_{\zeta}^{\omega}\right)^{\prime}(z) \omega(\zeta) d A(\zeta)$, and hence (2.1) gives

$$
\left|\left(P_{\omega}(h)^{\prime}(z)\right)\right| \leqslant\|h\|_{L^{\infty}} \int_{\mathbf{D}}\left|\left(B_{\zeta}^{\omega}\right)^{\prime}(z)\right| \omega(\zeta) d A(\zeta) \asymp \frac{\|h\|_{L^{\infty}}}{1-|\zeta|}, \quad|\zeta| \rightarrow 1^{-} .
$$

It follows that $P_{\omega}: L^{\infty} \rightarrow \mathcal{B}$ is bounded.

To see that $P_{\omega}: L^{\infty} \rightarrow \mathcal{B}$ is onto, let $g \in \mathcal{B}$. Then it induces an element in $\left(A_{\omega}^{1}\right)^{*}$ by the formula

$$
f \mapsto \lim _{r \rightarrow 1^{-}} \int_{\mathbf{D}} f_{r}(z) \overline{g(z)} \omega(z) d A(z)
$$


On the other hand, the Hahn-Banach theorem and the well-known duality $\left(L_{\omega}^{1}\right)^{*} \simeq$ $L^{\infty}$ guarantee the existence of $\varphi \in L^{\infty}$ such that

$\lim _{r \rightarrow 1^{-}} \int_{\mathbf{D}} f_{r}(z) \overline{g(z)} \omega(z) d A(z)=\int_{\mathbf{D}} f(z) \overline{\varphi(z)} \omega(z) d A(z)=\lim _{r \rightarrow 1^{-}} \int_{\mathbf{D}} f_{r}(z) \overline{\varphi(z)} \omega(z) d A(z)$

for all $f \in A_{\omega}^{1}$. Now, note that $P_{\omega}\left(f_{r}\right)=f_{r}$ and that $P_{\omega}$ is self-adjoint. We have

$$
\lim _{r \rightarrow 1^{-}} \int_{\mathbf{D}} f_{r}(z) \overline{g(z)} \omega(z) d A(z)=\lim _{r \rightarrow 1^{-}} \int_{\mathbf{D}} f_{r}(z) \overline{\left(P_{\omega}(\varphi)\right)(z)} \omega(z) d A(z) .
$$

But $P_{\omega}(\varphi) \in \mathcal{B}$ by the first part of the proof, thus $g-P_{\omega}(\varphi) \in \mathcal{B}$ and represents the zero functional. It follows that $g=P_{\omega}(\varphi)$, which completes the proof.

With these preparations we are ready to prove our main result.

\section{Proof of Theorem 1}

Let $F \in\left(A_{\omega}^{p}\right)^{*}$. Since $f_{r} \rightarrow f$ in $A_{\omega}^{p}$, we have $F\left(f_{r}\right) \rightarrow F(f)$. The weight $W$ induces a reproducing formula through its kernel $B_{z}^{W}$ by

$$
f_{r}(z)=\int_{\mathbf{D}} f_{r}(\zeta) \overline{B_{z}^{W}(\zeta)} W(\zeta) d A(\zeta), \quad z \in \mathbf{D}
$$

and hence (for instance, by approximating $f_{r}$ by polynomials) one obtains

$$
F\left(f_{r}\right)=\int_{\mathbf{D}} f_{r}(\zeta) F_{z}\left(\overline{B_{z}^{W}(\zeta)}\right) W(\zeta) d A(\zeta)
$$

Here the subindex in $F_{z}$ indicates the variable of the function with respect to which $F$ operates. Of course, by using any orthonormal basis $\left\{e_{n}\right\}$ of $A_{W}^{2}$, the kernel can be expressed as $B_{z}^{W}(\zeta)=\sum_{n} e_{n}(\zeta) \overline{e_{n}(z)}$, and hence one can explicitly write

$$
F\left(f_{r}\right)=\int_{\mathbf{D}} f_{r}(\zeta)\left(\sum_{n} \overline{e_{n}(\zeta)} F\left(e_{n}\right)\right) W(\zeta) d A(\zeta) .
$$

Anyhow, the function

$$
g(\zeta)=\overline{F_{z}\left(\overline{B_{z}^{W}(\zeta)}\right)}=\sum_{n} e_{n}(\zeta) \overline{F\left(e_{n}\right)}
$$

satisfies

$$
F\left(f_{r}\right)=\int_{\mathbf{D}} f_{r}(\zeta) \overline{g(\zeta)} W(\zeta) d A(\zeta)=\left\langle f_{r}, g\right\rangle_{A_{W}^{2}}
$$

Moreover, using the linearity of $F$ on the difference quotient along with the fact that for a fixed $\zeta \in \mathbf{D}, z \mapsto \overline{B_{z}^{W}(\zeta)}$ is analytic in a disc containing $\overline{\mathbf{D}}$, it is easy to see that $g \in \mathcal{H}(\mathbf{D})$ and

$$
g^{\prime}(\zeta)=\overline{F_{z}\left(\overline{\left(B_{z}^{W}\right)^{\prime}(\zeta)}\right)}, \quad \zeta \in \mathbf{D} .
$$

Therefore, since $\overline{B_{z}^{W}(\zeta)}=B_{\zeta}^{W}(z),(2.1)$ yields

$$
\begin{aligned}
\left|g^{\prime}(\zeta)\right|^{p} & =\left|\overline{\frac{d}{d \bar{\zeta}} F\left(B_{\zeta}^{W}\right)}\right|^{p}=\left|F\left(\frac{d}{d \bar{\zeta}} B_{\zeta}^{W}\right)\right|^{p} \leqslant\|F\|^{p}\left\|\frac{d}{d \bar{\zeta}} B_{\zeta}^{W}\right\|_{A_{\omega}^{p}}^{p} \\
& =\|F\|^{p} \int_{\mathbf{D}}\left|\frac{z}{\bar{\zeta}}\left(B_{\zeta}^{W}\right)^{\prime}(z)\right|^{p} \omega(z) d A(z) \leqslant \frac{\|F\|^{p}}{|\zeta|^{p}} \int_{\mathbf{D}}\left|\left(B_{\zeta}^{W}\right)^{\prime}(z)\right|^{p} \omega(z) d A(z) \\
& \lesssim\|F\|^{p} \int_{0}^{|\zeta|} \frac{\widehat{\omega}(t)}{\widehat{W}(t)^{p}(1-t)^{2 p}} d t=\|F\|^{p} \int_{0}^{|\zeta|} \frac{d t}{(1-t)^{1+p}} \asymp \frac{\|F\|^{p}}{(1-|\zeta|)^{p}}, \quad|\zeta| \rightarrow 1^{-},
\end{aligned}
$$


and it follows that $g \in \mathcal{B}$.

We next show that each $g \in \mathcal{B}$ induces a bounded linear functional on $A_{\omega}^{p}$ via $\langle\cdot, g\rangle_{A_{W}^{2}}$. To see this, we first show that $W \in \mathcal{D}$. A radial weight $W$ belongs to $\mathcal{D}$ if and only if there exist $C=C(W)>0, \alpha=\alpha(W)>0$ and $\beta=\beta(W) \geqslant \alpha$ such that

$$
C^{-1}\left(\frac{1-r}{1-t}\right)^{\alpha} \widehat{W}(t) \leqslant \widehat{W}(r) \leqslant C\left(\frac{1-r}{1-t}\right)^{\beta} \widehat{W}(t), \quad 0 \leqslant r \leqslant t<1 .
$$

It is easy to see that the right-hand inequality is equivalent to $W \in \widehat{\mathcal{D}}[6]$, and an analogous argument shows that the left-hand inequality is satisfied if and only if $W$ has the doubling property $\widehat{W}(r) \geqslant C \widehat{W}\left(1-\frac{1-r}{K}\right)$ for some $C, K>1$. Now that $\widehat{W}(r)=\widehat{\omega}(r)^{\frac{1}{p}}(1-r)^{\frac{1}{p}-1},(3.1)$ applied to $\omega \in \widehat{\mathcal{D}}$ implies

$$
\widehat{W}(r) \leqslant C^{\frac{1}{p}}\left(\frac{1-r}{1-t}\right)^{\frac{\beta}{p}+\frac{1}{p}-1} \widehat{W}(t), \quad 0 \leqslant r \leqslant t<1,
$$

for some $C=C(\omega)>0$, and hence $W \in \widehat{\mathcal{D}}$. Moreover, for each $K>1$,

$$
\begin{aligned}
\widehat{W}(r) & \geqslant \widehat{\omega}\left(1-\frac{1-r}{K}\right)\left(1-\left(1-\frac{1-r}{K}\right)\right)^{\frac{1}{p}-1}\left(\frac{1-r}{1-\left(1-\frac{1-r}{K}\right)}\right)^{\frac{1}{p}-1} \\
& =\widehat{W}\left(1-\frac{1-r}{K}\right) K^{\frac{1}{p}-1}, \quad 0<r<1,
\end{aligned}
$$

and thus $W \in \mathcal{D}$.

Since $P_{W}: L^{\infty} \rightarrow \mathcal{B}$ is bounded and onto by Lemma 2, the open mapping theorem ensures the existence of $M=M(W)>0$ such that for each $g \in \mathcal{B}$ there is $h \in L^{\infty}$ for which $g=P_{W}(h)$ and $\|h\|_{L^{\infty}} \leqslant M\|g\|_{\mathcal{B}}$. Fubini's theorem shows

$$
\begin{aligned}
\int_{\mathbf{D}} f_{r}(z) \overline{g(z)} W(z) d A(z) & =\int_{\mathbf{D}} f_{r}(z) \overline{P_{W}(h)(z)} W(z) d A(z) \\
& =\int_{\mathbf{D}} f_{r}(z) \overline{\int_{\mathbf{D}} h(\zeta) \overline{B_{z}^{W}(\zeta)} W(\zeta) d A(\zeta)} W(z) d A(z) \\
& =\int_{\mathbf{D}} \overline{h(\zeta)}\left(\int_{\mathbf{D}} f_{r}(z) B_{z}^{W}(\zeta) W(z) d A(z)\right) W(\zeta) d A(\zeta) \\
& =\int_{\mathbf{D}} \overline{h(\zeta)}\left(\int_{\mathbf{D}} f_{r}(z) \overline{B_{\zeta}^{W}(z)} W(z) d A(z)\right) W(\zeta) d A(\zeta) \\
& =\int_{\mathbf{D}} f_{r}(\zeta) \overline{h(\zeta)} W(\zeta) d A(\zeta),
\end{aligned}
$$

and hence, as the $L^{1}$-mean $M_{1}(r, f)$ of $f \in \mathcal{H}(\mathbf{D})$ is increasing in $r$,

$$
\left|\langle f, g\rangle_{A_{W}^{2}}\right| \leqslant \lim _{r \rightarrow 1^{-}} \int_{\mathbf{D}}\left|f_{r}(\zeta)\right||h(\zeta)| W(\zeta) d A(\zeta) \leqslant\|h\|_{L^{\infty}}\|f\|_{A_{W}^{1}} \leqslant M\|g\|_{\mathcal{B}}\|f\|_{A_{W}^{1}} .
$$

Now that $W(S) \asymp \omega(S)^{\frac{1}{p}}$ for each Carleson square $S$ by the identity $\widehat{W}(r)=$ $\widehat{\omega}(r)^{\frac{1}{p}}(1-r)^{\frac{1}{p}-1}$, the measure $W d A$ is a 1 -Carleson measure for $A_{\omega}^{p}$ by [4, Theorem 1], and hence we deduce $\left|\langle f, g\rangle_{A_{W}^{2}}\right| \lesssim\|g\|_{\mathcal{B}}\|f\|_{A_{w}^{p}}$.

The explicit use of the surjectivity of $P_{W}: L^{\infty} \rightarrow \mathcal{B}$ can be avoided by arguing in the following way. First, use Green's theorem to the moduli of monomials or calculate 
directly to get

$$
\begin{aligned}
\left|\langle f, g\rangle_{A_{W}^{2}}\right| & \lesssim\left|\left\langle f^{\prime}, g^{\prime}\right\rangle_{A_{W^{\star}}^{2}}\right|+|f(0)||g(0)| \\
& \lesssim \lim _{r \rightarrow 1^{-}} \int_{\mathbf{D}}\left|f_{r}^{\prime}(z)\left\|g^{\prime}(z) \mid W^{\star}(z) d A(z)+\right\| f\left\|_{A_{\omega}^{p}}\right\| g \|_{\mathcal{B}}\right. \\
& \lesssim\|g\|_{\mathcal{B}} \lim _{r \rightarrow 1^{-}} \int_{\mathbf{D}}\left|f_{r}^{\prime}(z)\right| \frac{W^{\star}(z)}{1-|z|} d A(z)+\|f\|_{A_{\omega}^{p}}\|g\|_{\mathcal{B}},
\end{aligned}
$$

where $W^{\star}(z)=\int_{|z|}^{1} \log \frac{s}{|z|} \omega(s) s d s$. Since the Cauchy integral formula and Fubini's theorem give $M_{1}\left(r, g^{\prime}\right)(1-r) \leqslant 4 M_{1}\left(\frac{1+r}{2}, g\right)$ for all $g \in \mathcal{H}(\mathbf{D})$ and $0<r<1$, and $W^{\star}(r) \asymp \widehat{W}(r)(1-r)$ for each radial weight $W$ and all $\frac{1}{2} \leqslant r<1$, a change of variable and the hypothesis $W \in \widehat{\mathcal{D}}$ yield

$$
\begin{aligned}
\left|\langle f, g\rangle_{A_{W}^{2}}\right| & \lesssim\|g\|_{\mathcal{B}} \int_{\mathbf{D}}\left|f^{\prime}(z)\right| \widehat{W}(z) d A(z)+\|f\|_{A_{\omega}^{p}}\|g\|_{\mathcal{B}} \\
& \lesssim\|g\|_{\mathcal{B}} \int_{\mathbf{D}}|f(z)| \frac{\widehat{W}(z)}{1-|z|} d A(z)+\|f\|_{A_{\omega}^{p}}\|g\|_{\mathcal{B}} .
\end{aligned}
$$

The assertion now follows similarly as above once we have shown that $\widehat{W}(z)(1-$ $|z|)^{-1} d A(z)$ is a 1 -Carleson measure for $A_{\omega}^{p}$. But since $W \in \mathcal{D}$, by (3.1) there exist $C=C(\omega)>0$ and $\alpha=\alpha(W)>0$ such that

$$
\int_{r}^{1} \frac{\widehat{W}(s)}{1-s} d s \leqslant C \frac{\widehat{W}(r)}{(1-r)^{\alpha}} \int_{r}^{1} \frac{d s}{(1-s)^{1-\alpha}} \lesssim \widehat{W}(r), \quad 0 \leqslant r<1,
$$

and the desired property follows by [4, Theorem 1]. One may also complete the proof directly from (3.2) by applying a result concerning differentiation operators $[4$, Theorem 2].

\section{References}

[1] Coifman, R., and R. Rochberg: Representation theorems for holomorphic and harmonic functions in $L^{p}$. - In: Representation theorems for Hardy spaces, Astérisque 77, 1980, 11-66.

[2] Forelli, F., and W. Rudin: Projections on spaces of holomorphic functions in balls. - Indiana Univ. Math. J. 24, 1974/75, 593-602.

[3] PelÁez, J.A., and J. RÄTtyä: Weighted Bergman spaces induced by rapidly increasing weights. - Mem. Amer. Math. Soc. 227:1066, 2014.

[4] PelÁez, J. A., and J. RÄTtyä: Embedding theorems for Bergman spaces via harmonic analysis. - Math. Ann. 362:1-2, 2015, 205-239.

[5] Peláez, J. A., and J. RÄttyÄ: Two weight inequality for Bergman projection. - J. Math. Pures Appl. 105, 2016, 102-130.

[6] Peláez, J.A., and J. RÄTtyä: On the boundedness of Bergman projection. - Advanced Courses of Mathematical Analysis VI, 2016, 113-132.

[7] Peláez, J. A., and J. RÄTtyä: Weighted Bergman projection on $L^{\infty}$. - Preprint.

[8] Romberg, B. W.: The $H^{p}$ spaces with $0<p<1$. - Ph.D. Thesis, University of Rochester, 1960.

[9] Shapiro, J.: Mackey topologies, reproducing kernels, and diagonal maps on the Hardy and Bergman spaces. - Duke Math. J. 43:1, 1976, 187-202.

[10] Zhu, K.: Bergman and Hardy spaces with small exponents. - Pacific J. Math. 162:1, 1994, 189-199. 